\title{
Predicting use of case management support services for adolescents and adults living in community following brain injury: A longitudinal Canadian database study with implications for life care planning
}

\author{
B. Baptiste ${ }^{\mathrm{a}, \mathrm{b}, \mathrm{c}, *}$, D.R. Dawson ${ }^{\mathrm{a}, \mathrm{c}, \mathrm{d}}$ and D. Streiner ${ }^{\mathrm{e}}$ \\ ${ }^{a}$ Department of Occupational Science \& Occupational Therapy, University of Toronto, Toronto, Canada \\ ${ }^{\mathrm{b}}$ Rehabilitation Management Inc., Toronto, Canada \\ ${ }^{\mathrm{c}}$ Graduate Department of Rehabilitation Science, University of Toronto, Toronto, Canada \\ ${ }^{\mathrm{d}}$ Rotman Research Institute, Baycrest, Toronto, Canada \\ ${ }^{\mathrm{e}}$ Department of Psychiatry, University of Toronto, Toronto, Canada
}

\begin{abstract}
.
OBJECTIVE: To determine factors associated with case management (CM) service use in people with traumatic brain injury (TBI), using a published model for service use.

DESIGN: A retrospective cohort, with nested case-control design. Correlational and logistic regression analyses of questionnaires from a longitudinal community data base.

STUDY SAMPLE: Questionnaires of 203 users of CM services and 273 non-users, complete for all outcome and predictor variables. Individuals with TBI, 15 years of age and older. Out of a dataset of 1,960 questionnaires, 476 met the inclusion criteria. METHODOLOGY: Eight predictor variables and one outcome variable (use or non-use of the service). Predictor variables considered the framework of the Behaviour Model of Health Service Use (BMHSU); specifically, pre-disposing, need and enabling factor groups as these relate to health service use and access.

RESULTS: Analyses revealed significant differences between users and non-users of CM services. In particular, users were significantly younger than non-users as the older the person the less likely to use the service. Also, users had less education and more severe activity limitations and lower community integration. Persons living alone are less likely to use case management. Funding groups also significantly impact users.
\end{abstract}

\footnotetext{
*Address for correspondence: Barbara D. Baptiste-M. Sc., Rehabilitation Science, Certified Life Care Planner, Rehabilitation Management Inc., 480 University Avenue, Suite 1008, Toronto, ON, Canada. Tel.: +416 365 0010; Fax: +416 365 1176; E-mail: barbara.baptiste@utoronto.ca.
} 
CONCLUSIONS: This study advances an empirical understanding of equity of access to health services usage in the practice of CM for persons living with TBI as a fairly new area of research, and considers direct relevance to Life Care Planning (LCP). Many life care planers are CM and the genesis of LCP is CM. The findings relate to health service use and access, rather than health outcomes. These findings may assist with development of a modified model for prediction of use to advance future cost of care predictions.

Keywords: Service use, prediction, brain injury, database, case management

\section{Introduction}

Case management and life care planning are associated health care sub-specialties focused on the development of current and future plans, including costs, for individuals with complex care needs. Traumatic brain injury (TBI) is widely acknowledged as a major, unresolved public health issue for adolescents and adults. Care needs are complex and evolving research points to TBI as a lifelong health condition (Masel \& DeWitt, 2010); thus, it is significant for those working in life care planning and case management. The estimated rate of TBI occurrence in the USA and Canada is in the range of 120 per 100,000 per year, excluding highest and lowest rates from the estimates, and is 15 times more common than spinal cord injury (Kraus \& Chu, 2005; Cochrane et al., 2000; Ontario Brain Injury Association [OBIA], 2012). In 2014 the Public Health Agency of Canada reported that, based on current data, the number of hospitalizations for TBI in Canada will increase $28 \%$ by 2031. Case Management (CM) has emerged as a significant health service over the past two decades (Applebaum \& White, 2000; Fitzsimmons, 2003; Quinn, Pannone, Gruman \& Roja, 2004), as has the sub-specialty of Life Care Planning (Masel \& DeWitt, 2010; Deutsch \& Sawyer, 1985; Klinger, Baptiste \& Adams, 2004). In 2009 National Standards of Practice were identified in Canada, and $\mathrm{CM}$ was defined as " ... a collaborative, client-driven process for the provision of quality health and support services through the effective and efficient use of resources. Case Management supports the clients' achievement of safe, realistic, and reasonable goals within a complex health, social, and fiscal environment" (National Case Management Network [NCMN], 2009). Also in 2009, the International Association of Rehabilitation Providers (IARP) produced Standards of Practice in Life Care Planning and a Life Care Plan (LCP) was defined as “. .. a dynamic document based upon published standards of practice, comprehensive assessment, data analysis and research, which provides an organized, concise plan for current and future needs with associated cost for individuals who have experienced catastrophic injury or have chronic health care needs" (Weed \& Berens, 2010, p. 3). Thus, both roles are specialty areas of health service support and rely on an expertise in planning for support, considering cost factors, in matters of complex care needs whether short-term or long term.

The practices of $\mathrm{CM}$ and LCPing occur within the context of varied health delivery systems and populations, including individuals with TBI. In their 2002 survey of service needs for persons with TBI, Heinemann et al., concluded that resource and service coordination was an important need for persons with TBI, and that a research-based understanding of how community-based services can be delivered to persons with TBI was necessary. Databases that assist in understanding the needs of persons living with TBI and their caregivers by collecting data directly from the source are considered an important contribution to research (Abreu et al., 2001).

Developing empirical understandings of access to health services and service use, in relation to care planning following TBI, is a new area of research. While there have been recent studies specifically focused on health services that are used following TBI (OBIA, 2012; Laursen \& Helweg-Larsen, 2012; Drag, Renninger, King, \& Hoblyn, 2012; Bland, Zampieri, \& Damiano, 2011) these do not include measureable factors associated with use and access; that is, identifying the elements that contribute to use and access of the services. Previous studies have examined immediate service use (Phillips, Greenspan, Stringer, Stoble, \& Lehtonen, 2004), and these highlight the need for further TBI service utilization studies. However, these studies focused on hospital-based rehabilitation services (PT, OT and ST) and immediate discharge, and not community-based services and needs. Canadian studies have looked at access and quality of primary care for 
people with disabilities (McColl et al., 2010) and access limitations for TBI (Ouellet, Sirois, \& Lavoie, 2009); however North American studies have not looked at the factors that may impact parity of access and thus, use of a service. International study on equity of service access following TBI, using the Andersen model of health service use (also referred to as the Behaviour Model of Health Service Use (BMHSU)), has been conducted in the Netherlands (Willemse-van Son, Ribbers, Stam, \& Van den Bos, 2009) and has been used to predict discharge destination following BI (Chen et al., 2012). However, to date, studies have neither focused on Canada nor the specific services of CM and LCPing. As $\mathrm{CM}$ is the clinical support that plans, coordinates and advocates for service access, both in community and in facilities, it is considered an important area of research study. Concomitantly, LCPing most often applies the experience base of CM in order to project support over a life span, and experienced CMs are the most likely practitioners to progress into LCPing (Weed \& Berens, 2010), the sequential relationship enables use of available data to study factors associated with access to $\mathrm{CM}$ to serve as a proxy for LCPing. To our knowledge, there are no databases at present that collect data on LCPing as a service use.

This study had two main objectives. The first objective was to describe the differences between a group of persons with traumatic brain injury who had used $\mathrm{CM}$ services, and a group who had not used CM services. The second objective was to determine factors associated with CM service use in people with TBI using a published framework of predisposing, need, and enabling factors (Aday \& Andersen, 1974; Andersen, Davidson, \& Ganz, 1994). This framework is known as the "Andersen Model" and also as the "Behaviour Model of Health Service Use" (BMHSU). The framework has been used in other Canadian studies in predicting factors for discharge location following $\mathrm{ABI}$ and entrance into nursing facilities for the ageing population (Chen et al., 2012; Tomiak et al., 2000; Cohen, Noralou, DeCoster, Black, \& Decker, 1995).. In a review of the model's development over a 25 year period, and with regard to its continued relevance, Andersen stated, "Its purpose is to discover conditions that either facilitate or impede utilization." (Andersen, 1995, p. 4). Similar to the World Health Organization's (WHO) conceptual model for health outcomes, International Classification of Function (ICF), the BMHSU uses "contextual factors". The purpose of the Andersen model has been to emphasize community, including the process and structure of healthcare, while ultimately focusing on the individual and their use of health services. This is particularly valuable for this study, due to a focus on the "individual characteristics" of service use within community. In a systematic review of the Behavioural Model of health Service use the authors concluded that the Andersen model, and variations, is widely acknowledged as the most well known and most used model of access to care (Goldsmith, 2002).

\section{Methods}

\subsection{Design}

A retrospective cohort study, with a nested casecontrol design, was used. Adolescents and adults who received case management services (users) were compared to those who did not receive the services (non-users). Factors associated with service use, applying the Andersen's Behavior Model of Health Services Use (BMHSU) (Aday \& Andersen, 1974; Andersen \& Davidson, 2001; Anderson, 1995). BMHSU, were analyzed, using a longitudinal, provincial database.

\subsection{Data collection}

The Caregiver Information and Support Link (CISL) longitudinal database provided the data for this study. This database was developed in 1989 by the Ontario Brain Injury Association (OBIA), to facilitate gathering data about the long-term needs of people with acquired brain injury (ABI) living in the community, including the personal cost of survivors and caregivers, through the use of a standardized questionnaire (OBIA, 2012). The questionnaire was designed to be administered annually to study participants. Longitudinal data from initial and follow-up questionnaires were used over a 13 year period from inception.

\subsubsection{The questionnaire}

The CISL questionnaire is distributed by OBIA contacts at Ontario hospitals, facilities, community associations, and provider groups working with people with $\mathrm{ABI}$; the questionnaire may also be provided to persons who contact OBIA directly (OBIA, 2013). Questionnaires may be completed by the person with brain injury, a family member, or "other". The adult version of the questionnaire (persons 15 and older) consists of 82 items spread across six categories: Descriptive Information; Classification of Injury; Activity Limitations; Community Integration; Service Use and Need; 
and Caregiver Stress. The question on CM falls under the Service Use and Need section of the questionnaire and defines $\mathrm{CM}$ as: "paid assistance with negotiating access to service". This study research uses CM both independently and as a proxy for LCPing as the latter is the plan for future service access and is often completed by case managers with expertise in long term planning following neurotrauma. As the CISL deals with community (post-acute) service data, it does not reflect services that may have been provided in the early (acute and subacute) stages of recovery when many of the participants would likely have been hospitalized.

\subsubsection{Derivation of the study sample}

The initial questionnaires are filled out upon first contact with the organization, and follow up is according to annual contact by the organization, with permission of the respondent or their proxy. The CISL questionnaire data were input into a Paradox database by an OBIA employee. At a subsequent point another OBIA employee verified the entrees against a paper copy of the questionnaire. All personal identifiers were removed from the study data. Questionnaires in the CISL database were reviewed for the following criteria:

1. Respondents had a traumatic brain injury. This was determined by responses to the "Injury Type" question of the questionnaire. Respondents who indicated their injuries resulted from the following circumstances were included in the study sample: driver of an auto in an accident, passenger of an auto in an accident, motorcycle, pedestrian struck by an auto, bicyclist struck by an auto, bicycle fall (no auto involved), victim of assault, fall (not while employed), accident at work, recreational vehicle crash, and sports injury. The option "other" in the CISL questionnaire was not included in the sample because it included sources of injury that lacked clarity for analysis.

2. Respondents were at least 15 years old at the time of completion of either an initial or follow-up questionnaire. Thus they fall in the category of adolescents, as well as adults.

3. The questionnaire was complete for the nine variables used in the analysis. These variables are described in the following section.

All available initial and follow-up questionnaires for each respondent that met these criteria were reviewed to determine the respondent's case group. The first time a respondent self-identified as a CM service user, this questionnaire was included in the positive case group ("Y", in Diagram 1), but his or her subsequent questionnaires were not included in the data set. If a respondent never indicated that he or she used CM services, the last available questionnaire was used in the data analyses to ensure accuracy. The conceptual framework for capturing this data is provided in Fig. 1.
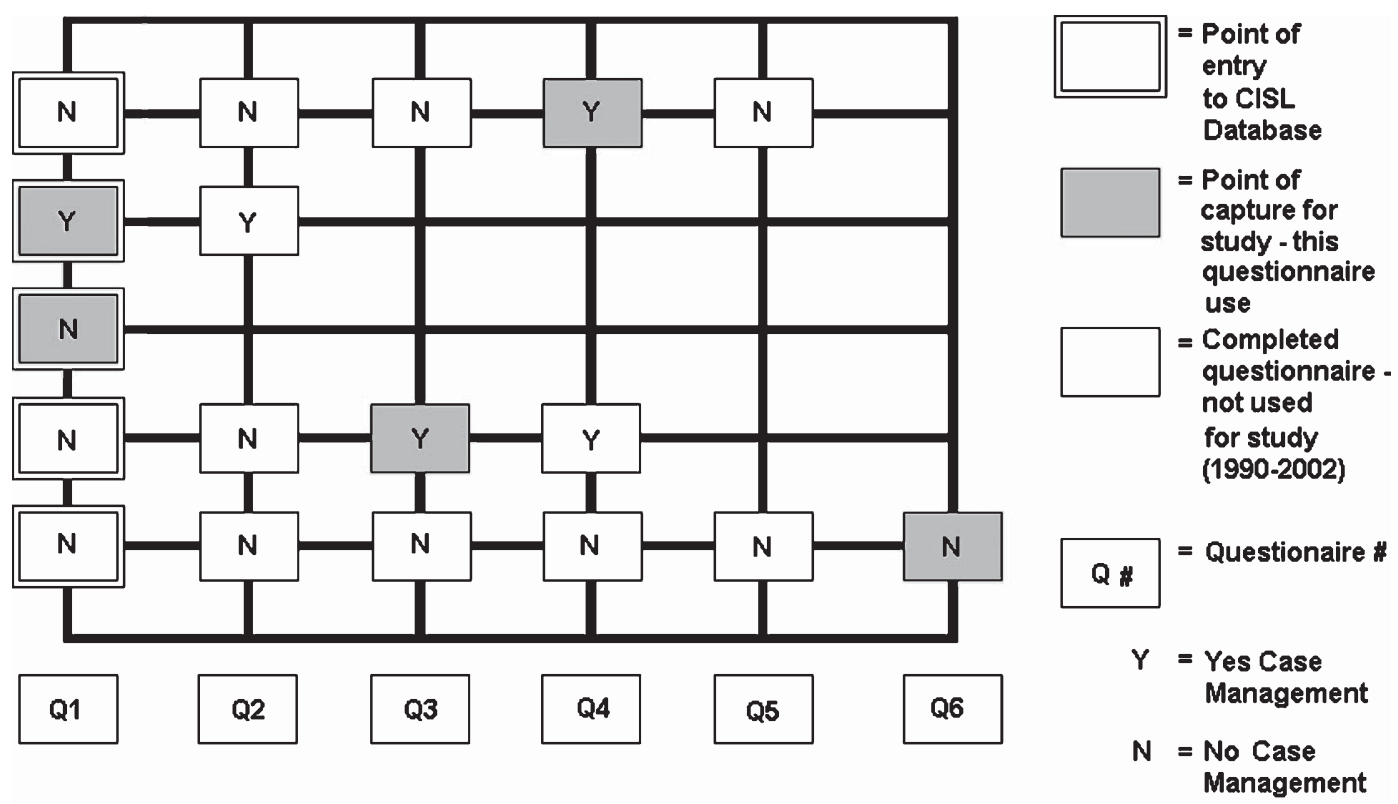

Fig. 1. Conceptual drawing of selection strategy for case (Y) and control groups (N). 


\subsubsection{Measures}

One outcome variable (CM service use) and eight predictor variables (see factors below) were grouped using Andersen's Behavior Model of Health Services Use (BMHSU) (Aday \& Andersen, 1974; Andersen \& Davidson, 2001; Anderson, 1995). These predictors are: pre-disposing, need and enabling factors.

CM Service Use Questionnaire respondents indicated whether or not they had used CM services in the previous month; this was the outcome variable. Use of $\mathrm{CM}$ services was coded as 1 , non-use was coded as 0 .

Predisposing Factors Information was collected on the respondents' age, gender, and pre-injury education level. Age at injury, in years. Male gender was coded as 0 , female gender as 1 . Similarly, for education before injury, high school education or less than high school education was coded as 0 , and any postsecondary education was coded as 1 .

Need Factors The portions of the questionnaires regarding the severity of injury, activity limitations, and community integration provided the data about the potential need for $\mathrm{CM}$ services. Severity of injury was determined by the length of coma (LOC) in hours. Activity limitations were measured using the composite score from the Activity Limitations Index of the CISL questionnaire. This is a continuous variable with a maximum score of 41 ; higher scores indicate higher or more limitations. Community integration was measured using the 15-item Community Integration Questionnaire (CIQ), with a maximum score of 27 . Higher scores on the CIQ indicate more integration. These activity and community integration factors are similar to the International Classification of Function (ICF) (World Health Organization, 2014) with regard to activity and participation factors.

Enabling Factors The source of funding for CM services, and the respondents' living arrangements were considered enabling factors. The source of funding was coded as the most likely source based on the type of injury. Motor Vehicle Collisions/Auto Insurance was the most likely source of funding for respondents who chose the following injury types: driver of auto; passenger of an auto accident; motorcycle; pedestrian struck by an auto; bicycle struck by an auto; or recreational vehicle crash. This funding source was coded as 0 . Work/Workplace Safety and Insurance Board funding was coded as 1 and used for respondents who were injured in an accident while at work. Respondents injured in a bicycle fall (no auto involved), fall (not while employed), sports injury, or as a victim of assault were included in the "other" funding category, coded as 2 . If respondents selected more than one cause of injury, a single cause was assigned based on hierarchy of significance. For example, if the respondent checked "other", but explained that the injury occurred at work, the response was re-classified as "accident at work". Present living arrangement was also a polychotomous variable with three categories: living at home with family; living at home alone; or living in a facility. Living at home with family, coded as 1, included responses of "own family/parents", "own family/spouse", and “own family/brother or sister". Living at home alone, coded as 2 , included responses of "independent", "board" and "single room occupancy". Living in a facility, coded as 3 , included responses of "care/foster care", "acute care hospital", "inpatient rehabilitation hospital", "transitional living centre", "skilled nursing facility", "inpatient psychiatric centre", "intermediate care facility", "supervised community residence", "intensive community residence", and "supportive community residence".

\subsection{Data analysis}

\subsubsection{Descriptive analyses}

To determine the differences between CM users and non-users, chi-square tests were used for categorical variables (gender, pre-injury education, living arrangements, and funding source), and independent sample $t$-tests were used for continuous variables (age, severity of injury, activity limitations, and community integration). Predictor variables were then examined for collinearity. Relational concerns were addressed using Pearson correlation coefficients for continuous variables and Spearman's rho coefficients for categorical variables. Most correlations were modest, with the highest correlation between the ALI and CIQ $(r=0.43)$.

Sequential (hierarchical) logistic regression was used to create a model predicting CM use. The order in which the groups of variables - predisposing factors first, need factors second, and enablement factors last - were entered into the model was derived from Andersen and Davidson's (2001) BMHSU. They suggest that need, as opposed to available resources, will explain most of the variance in health service use. Wald tests were used to determine the statistical significance of each regression coefficient in the model. Data were entered into the SPSS statistical software package, version 11.0 which solves for the group coded 1 (i.e., CM users). 
Table 1

Study sample $(N=476)$ by predictor variables and user groups

\begin{tabular}{lcc}
\hline & $\begin{array}{c}\text { No CM Use } \\
(N=273)\end{array}$ & $\begin{array}{c}\text { Yes CM Use } \\
(N=203)\end{array}$ \\
\hline $\begin{array}{l}\text { Pre-disposing Factors } \\
\text { Age at injury (M, SD) }\end{array}$ & $41.18(12.19)$ & $35.67(13.51)^{*}$ \\
Gender & 67.03 & 67.98 \\
- \% Male & 32.97 & 32.02 \\
- \% Female & & \\
Education prior to injury & 55.31 & 51.72 \\
- \% with HS or less & 44.69 & 48.28 \\
- \% with College/University & & \\
Need Factors & & \\
LOC in hours & 16.85 & $6.40^{* *}$ \\
- \% Mild/Moderate & 83.15 & 93.60 \\
- \% Severe & $15.78(8.76)$ & $18.40(8.45)^{* *}$ \\
ALI (M, SD) & $17.15(5.37)$ & $15.43(4.94)^{*}$ \\
CIQ (M, SD) & & \\
Enabling Factors & & \\
Source of Funding & 75.09 & $86.21^{* *}$ \\
- \% Auto insurance & 6.59 & 3.94 \\
- \% Work insurance & 18.32 & 9.85 \\
- \% Other & & \\
Present Living Arrangement \pm & 62.30 & $62.60^{*}$ \\
- \% Home with family & 28.20 & 12.30 \\
- \% Home alone & 9.50 & 25.10 \\
- \% Facility & & \\
\hline
\end{tabular}

LOC = length of coma, ALI = Activity Limitations Index, CIQ = Community Integration Questionnaire. *significant at $p<0.001$. **significant at $p<0.05$.

\section{Results}

From this pool of questionnaires, the inclusion criteria and selection strategy yielded a sample of 476 questionnaires.

Note: Pearson $r$ coefficients are reported for continuous variables, spearman $r$ for categorical and point biserial for all variables. Correlations with $p$-values of 0.01 or less are in bold.

The CM-user group consists of 203 respondents, and the remaining 273 respondents were non-users. The demographic characteristics of the sample are provided in Table 1, according to the BMHSU.

\subsection{Differences between users and non-users}

Age: CM users in the study sample had a mean age of 35.67 years ( $\mathrm{SD}=13.51$ ), which is significantly younger than the non-user group whose mean age was $41.18(\mathrm{SD}=12.19)$.

Gender: The ratio of males to females in both groups was similar. The non-user group was $67 \%$ male, and the user group was $68 \%$ male.

Pre-injury Education: The percent of respondents with high school education or less was similar for both groups. Approximately 55\% of non-users had a high school diploma or less, and approximately $52 \%$ of users had this level of education.

Severity of Injury: The CM user group experienced more severe injuries than the non-user group. The percentage of respondents in the user group whose coma lasted more than six hours was $93.6 \%$, compared to $83.2 \%$ of non-users.

Activity Limitations: The CM user group had a mean score on the Activity Limitations Index of 18.4. The non-user group had a significantly lower mean score, with 15.8 .

Community Integration: This measure was also statistically significant. The CM user group had a lower mean score (15.43) on the Community Integration Questionnaire than the non-user group (17.15).

Source of Funding: The CM user group received significantly more funding from auto insurance than the non-user group. In the non-user group, $75 \%$ of respondents received funding from auto insurance, compared to $86 \%$ in the user group.

Living Arrangements: The current living arrangements also differed between the groups. While a similar percentage of individuals in each group indicated they were living with family, the CM user group had a higher percentage of respondents living in a facility, but the non-user group had a higher percentage of respondents living at home alone.

\subsection{Correlational analyses}

Table 2 provides the correlation coefficients for each of the eight predictor variables. Most correlations were very low, with the highest correlation between Activity Limitations and Community Integration. The low correlations between variables indicated that there would

Table 2

Correlation matrices for continuous and categorical predictor variables

\begin{tabular}{lrcccccr}
\hline & Age CIQ & ALI & \multicolumn{3}{c}{ Gender } & Education & \multicolumn{3}{c}{ PLA } \\
\hline Age & $-\mathbf{0 . 1 4}$ & 0.10 & 0.09 & $\mathbf{0 . 2 1}$ & 0.01 & $\mathbf{0 . 1 8}$ & $\mathbf{- 0 . 2 3}$ \\
CIQ & & $\mathbf{0 . 4 3}$ & $\mathbf{0 . 1 5}$ & 0.01 & 0.05 & 0.09 & 0.03 \\
ALI & & & 0.06 & 0.05 & 0.02 & -0.05 & 0.10 \\
Gender & & & - & 0.08 & 0.00 & -0.10 & -0.11 \\
Education & & & & - & 0.04 & -0.04 & -0.06 \\
PLA & & & & & - & 0.04 & 0.06 \\
Funding & & & & & & - & $-\mathbf{0 . 1 4}$ \\
Coma & & & & & & & - \\
\hline
\end{tabular}

Correlations with $p$-values of 0.01 or less are in bold. Legend: $\mathrm{CIQ}=$ community integration questionnaire; $\mathrm{ALI}=$ activity limitation index; PLA = Living Arrangement. 
Table 3

Case management use as function of predisposing, needs and enabling factors

\begin{tabular}{|c|c|c|c|c|c|}
\hline \multirow[t]{2}{*}{ Variables } & \multirow[t]{2}{*}{$B$} & \multirow[t]{2}{*}{ Wald Test (z-ratio) } & \multirow[t]{2}{*}{ Odds Ratio } & \multicolumn{2}{|c|}{$\begin{array}{l}95 \% \text { Confidence Interval } \\
\text { for Odds Ratio }\end{array}$} \\
\hline & & & & Lower & Upper \\
\hline \multicolumn{6}{|l|}{ Pre-Disposing } \\
\hline Age & $-0.035^{*}$ & 16.05 & 0.966 & 0.949 & 0.982 \\
\hline Pre-Trauma Education & $-0.524^{* *}$ & 6.06 & 0.509 & 0.388 & 0.898 \\
\hline \multicolumn{6}{|l|}{ Need } \\
\hline LOC & $0.795^{* * *}$ & 4.952 & 0.451 & 0.224 & 0.910 \\
\hline ALI & $0.028^{* * *}$ & 4.516 & 1.028 & 1.002 & 1.055 \\
\hline CIQ & $-0.047^{* * *}$ & 4.094 & 0.954 & 0.911 & 0.999 \\
\hline \multicolumn{6}{|l|}{ Enabling } \\
\hline \multicolumn{6}{|l|}{ Living Arrangement ${ }^{\mathrm{a}}$} \\
\hline DC (1) home alone & $-0.561^{* * *}$ & 3.772 & 0.571 & 0.324 & 1.005 \\
\hline DC (2) facility & $0.952^{*}$ & 10.966 & 2.590 & 1.475 & 4.549 \\
\hline \multicolumn{6}{|l|}{ Funding Source $^{\circ}$} \\
\hline DC (1) Worker Insurance & -0.660 & 1.914 & 0.517 & 0.203 & 1.316 \\
\hline DC (2) Other & -0.620 & 3.923 & 0.538 & 0.291 & 0.994 \\
\hline
\end{tabular}

LOC = length of coma; ALI = Activity Limitation Index; CIQ = Community Integration Questionnaire; DC = Dummy Code. ${ }^{\mathrm{a}}$ living arrangement at time questionnaire completed; home with family (reference cell). ${ }^{\circ}$ automobile insurance (reference cell). ${ }^{*} p<0.05 ;{ }^{* *} p<0.01 ;{ }^{* * *} p<0.001$.

be no redundancy in the model (Tabachnick \& Fidell, 2001; Hosmer \& Lemeshow, 2000)

\subsection{Inferential analyses}

Hierarchical logistic regression analysis was performed to determine which factors predict the use of CM services. Table 3 shows the regression coefficients, Wald statistics, and odds ratios for seven of the eight predictor variables. Gender was not significant and did not add anything to the model; it was therefore removed from the model. A Goodness-of-fit test of the full model with the three predictor groups against a constant-only model was statistically significant, $X^{2}$ (degrees of freedom $\left.=10, N=476\right)=81.546$, $p=0.000$. Goodness-of-fit was also evaluated using the Hosmer-Lemeshow statistic. Results reveal good statistical reliability of the full model with all eight predictors at $X^{2}(8, N=476)=12.038$, with a $p$ equal to 0.150 . Using this statistic a good model produces a non-significant chi-square (Tabachnick \& Fidell, 2001; Hosmer \& Lemeshow, 2000).

According to the Wald criterion, age, education, severity of injury, activity limitations, community integration, living at home alone, living in a facility, and funding by "other" insurance all significantly and reliably predict the use of CM services. Using the Wald scores, age and living in a facility and other insurance had the largest individual contributions to the model.

The odds ratios provide important information about the degree of influence each variable has on the use of CM services. For each 1 year increase in age, CM service use is $5 \%$ less likely. Thus, the younger a person with traumatic brain injury is, the more likely he or she is to access CM services. Higher levels of education also decrease the likelihood of CM use. People with at least some college or university education were $40 \%$ less likely to use CM services. People with severe traumatic brain injury - coma lasting seven or more hours - are 55\% less likely to use CM than people whose coma lasted six hours or less. The likelihood of CM service use increases by $3 \%$ for each one-unit increase in the Activity Limitations Index score, therefore, people with more activity limitations are more likely to access $\mathrm{CM}$ services. Community integration is negatively correlated with $\mathrm{CM}$ service use. The use of $\mathrm{CM}$ is 6\% less likely for each one-unit increase on the Community Integration Questionnaire. People living in a facility are 2.5 times more likely to use CM services, but people living at home alone are $43 \%$ less likely to use CM. Finally, people whose expenses were funded by "other" insurance were $47 \%$ less likely to access CM services.

\section{Discussion}

Analyses revealed some significant differences between users and non-users of CM services. Users were significantly younger than non-users. Proportions of male to female, and persons with high school/less and more than high school education were the same for both groups. Persons with less than high school education were more likely to use $\mathrm{CM}$, which may be a 
function of age (as users were also younger). Case management non-users had less severe injuries, while users had significantly greater activity limitations and were significantly less integrated into the community. The $\mathrm{CM}$ users group was funded more through automobile insurance and was more likely to live in facilities or home with others than non-users, while non-users were more likely to live alone. These findings are potentially relevant as they raise questions about possible parity of access for persons based on age, community living status, and funding source: older persons, persons living alone and persons not insured by automobile insurance may not be receiving access. Overall, increased severity with regard to high scores on activity limitations as measured by the ALI, and low scores for participation in home, social and work domains, as measured by the CIQ, results in increased service use. Based on LOC, persons in this study fell into the moderatesevere range. Epidemiological study has pointed to the literature indicating that persons with mild TBI can be equally high service-users alongside persons with moderate and severe TBI (Kraus \& Chu, 2005); thus, this may be an area for further study.

Comparisons to other studies relating service use outcomes to functional capacity as exemplified in activity limitations and community re-integration were difficult because this research on service use, as an outcome variable, is new. Other service-use studies did not classify service use as an outcome, but rather considered the number of services used and effect on outcomes in the community (Hodgkinson, Veerabansa, Drane, \& McCluskey, 2000; Schootman \& Fuortes, 1999; Tennant, MacDermott, \& Neary, 1995). However, as the BMHSU informs (Anderson, 1995), availability of a service is not the same as equity of access. A service may be available, but a person may not be able to access (use) it. For example, they may not be aware of the service or it may require funding they do not know how to access. A survey study $(N=596)$ was commissioned through the Ontario Brain Injury Association (OBIA) in 2012 looking at the impact of services on individuals with brain injury, and this included CM (OBIA, 2012). $\mathrm{CM}$ was concluded to be a valued service by $88 \%$ of the respondents who received the support $(N=372)$; however, $50 \%$ of respondents said such services were not available to them.

Odds ratios using logistic regression analysis for predisposing factors revealed statistically significant findings for age and education. These were: (a) the older you are the less likely to have used CM; (b) the more education, the less likely to have used case management. Statistically significant findings of the need factors revealed that (b) CM use is less likely with persons with more severe TBI; (c) the greater a person's activity limitations the more likely they have used $\mathrm{CM}$; (d) case management was less likely to be used by persons who were more integrated into their community. Finally, with regard to enabling factors, statistically significant findings were (a) persons living alone were significantly less likely to use $\mathrm{CM}$ and persons living in facilities were more likely to use CM; and (b) persons funded by other insurance were less likely to have received $\mathrm{CM}$ services.

This was the first known study of the association between case management use and TBI, using a planned framework for health service-use and prediction; thus there are no comparative data. However, the findings appear consistent with health service utilization research that has examined health outcomes and service use and/or unmet services among persons with TBI. Implications are discussed using the planned order of predisposing, need and enabling factors.

First, older persons are less likely to use case management, with the corollary that younger persons are more likely to use this support. The association between age and CM use is relevant for varied reasons. It may reveal a lack of access to services for older persons, or perhaps a reduction in service use with aging. In the first scenario, if the delivery system is managed through the automobile insurance scheme, and older persons have less of a chance to return to work or be considered productive in society, then the cost containment benefits of case management are very limited, and in fact may increase costs by increasing service use for quality of life or functional improvement without an outcome of cost savings (i.e., a return to work). In other words, there is no financial incentive for the insurance company to fund the service. Also, litigation benefits are more limited with the elderly; therefore, there would be less of an opportunity for access to a lawyer or facilitator who may advocate for services. Similarly, if the delivery system is the Worker's Safety Insurance Board, an older person is less likely to be in this group because they have too few years of employment ahead and the cost/benefit ratio for $\mathrm{CM}$ use would be limited, or non-existent, with regard to return to work; alternatively, the person who sustained the TBI could be retired and simply would not be covered under this insurance scheme. Use of CM by the elderly would then rely on accessing public community care ("other" in my source of funding); however, odds ratio results reveal that persons covered by "other" insurance are $47 \%$ less likely to use CM services. This 
may in fact highlight a significant lack of services for relatively older persons in the public sector. It is a public health issue, as the recent report (Public Health Agency of Canada, 2014) predicts the number of Canadians age 65 years and older; living with TBI, will more than double by 2031. This is important for life care planning to ensure that supports typically high when a person is young, need to be considered for increase as they age due to lack of access. Caution must be used in interpreting this as this is new research and there are constraints to the data; however, further analysis is warranted, as these findings reveal an association between aging and lack of access to a service. This speaks to the Andersen's concept of equity (Andersen \& Davidson, 2001).

Findings on the predictor of education reveal that the more educated a person is before sustaining the TBI, the less likely to use case management. Age and education were only moderately correlated $(r=0.21)$, therefore only some of the variance may be associated with age and the rest with education. While this appears somewhat counter-intuitive there are varied possibilities, such as better self-advocacy by those with more education or ability to independently access services, these persons are better educated about their injury, or perhaps have better access to support networks. Also, it may be that the adolescents, who have not yet finished high school, are receiving access to CM services. The implications are not clear however it is an interesting finding, and may or may not be particularly relevant. Long-term studies of service use and TBI are limited, as there are also few studies available that report on unmet needs (Heinemann, Sokol, Garvin, \& Bode, 2002) and long-term use of services and socio-demographic characteristics (Colantonio et al., 2004); however, these do not report on the association of socio-demographic factors and the use of case managed services. Overall, the findings reveal a need for further understanding of issues of service access, use and need associated with predisposing factors, particularly age and education, and TBI. This was similar to the findings of (Willemse-van Son et al., 2009).

In the factor group of need, results on injury severity as defined by LOC, reveal that CM use is $55 \%$ less likely the more severe the participant. This appears counterintuitive and may be related to the highly skewed data on LOC. The other two measures of need - activity limitations and community integration - predicted higher service use. Greater activity limitations and less community integration result in a significant increased use of CM. This appears to be clinically sound; however, it does raise an important question about persons with mild traumatic brain injury (MTBI) who were minimally represented in the study sample, and whose identification within health databases and whose service needs have been neglected in the extant literature (Van Holst, Cassidy, 2004). My findings reveal that there is a need to represent this group and understand their service use and needs. This finding also may point to the value of functional data (activity and participation) for prediction of service use.

The findings from analysis of the two enabling factors reveal that participants living alone are significantly less likely to use CM, and that use of CM was significantly more likely if a participant lived in a facility. In the former living arrangement (alone), concerns about marginalization for a single person living with TBI may be raised and further analysis of this factor warranted. The finding that living in a facility increases the odds of using $\mathrm{CM}$ is curious, especially since earlier findings revealed that those participants with more severe injuries are less likely to use CM, and since persons with more severe injuries would be expected to be those participants living in a facility. However, as OBIA has access to persons living within community facilities, this case group may have access to facility-based CM services. Living at home with family was a reference cell for the regression analysis as it was the most frequently occurring living arrangement (60\%). Due to the age range of the study participants (starting at 15 years of age) marriage was not analysed; however, living at home with family was considered a proxy for support levels.

It appears that participants whose source of injury, and therefore funding, is categorized as "other" (public insurance and possible employer insurance - Long Term Disability or Canada Pension Plan), and who are not covered by auto or worker insurance, are less likely to use CM. This may reflect fiscal and policy restrictions of these systems, or less serious injuries or limited advocacy for these groups. The findings may also indicate that inequities may exist between insurance schemes. Findings from a study conducted in Kentucky $(N=3,821)$ (Svenson \& Spurlock, 2001) revealed that insurance status was significantly associated with admission for hospital services for persons with head injury. Further study of this particular predictor may be valuable in understanding equity of access in Canada.

In summary, the largest individual contributions to the prediction of $\mathrm{CM}$ service use were age and living arrangements. In addition, education, severity of injury, activity limitations, participation restrictions and 
funding source, were all significantly associated with $\mathrm{CM}$ use, and contributed to the analytical model. This information may help clinicians involved in analysis of long-term service use needs of their clients with TBI, as well as providers, funders, policy-makers and administrators who need to project service use and enable access to supports. In addition, the findings may help us to better understand issues of service access, including parity of access. This is important for consumers, caregivers and all persons living and working with persons with TBI.

While the reliability of the sequential, planned predictors was not a specific study objective, it is worthwhile to note that these preliminary findings indicate that predisposing, need and enabling factors were reliable predictors of $\mathrm{CM}$ service use in a sequential fashion, with each factor group contributing to the overall multivariate model. Gender did not reliably enhance prediction; however, all other factors reliably predicted service use. These findings may assist with development of a modified model, based on the BHMSU, for prediction of use and eventually future cost of care following trauma.

\subsection{Study strengths and limitations}

Studies on the prediction of CM service use by persons with TBI do not exist in the medical/rehabilitation literature, and neither does an established method for predicting service use following TBI. In this study we have tried to frame such a method by using a published model based on significant health service research and extensive theoretical considerations (Forbes \& Janzen, 2004; Tomiak, Berthelot, Guimond \& Mustard, 2000). The CISL sample was self-selected and self-reported. Selection bias, particularly impacting on bias towards the null, and measurement bias, in particular response and recall bias, may possibly impact on the validity of findings. These limit the generalizability of the study and caution is warranted in the interpretation of the findings.

In 2004, Fitzsimmons reported on 51 case studies with case management intervention for persons with TBI, acknowledging brain injury case management as a "comparatively new discipline" (Fitzsimmons, 2003, p. 947). The lack of homogeneity with definitions also creates a problem (Quinn et al., 2004). This study has attempted to elucidate some factors associated with the use of CM services using a parsimonious approach based on both the conceptual framework to choose predictor variables and the statistical analysis of multiple logistic regression which works best when the principle of parsimony is applied (Hosmer \& Lemeshow, 2000). There are numerous factors that may influence service use, and variations in service utilization across studies may be partly explained by different health systems and partly by varying $\mathrm{CM}$ delivery models. In addition, unmet service needs (whether perceived, evaluated or realized) and unfelt needs, that is, services which individuals are unaware of, thus reducing activities, participation and perceived need, (Heinemann, Corrigan, \& Moore, 2004) require further investigation and were not explored in this study.

A potentially significant factor about use of case managed services by person with TBI, that this study was not able to capture, was service availability in a person's region of residence; in particular, urban as compared to rural environments. In the 2012 OBIA survey study (OBIA, 2012), of the $33 \%$ of respondents who did not receive $\mathrm{CM}, 50 \%(N=167)$ said that such services were not available to them. Thus, this is an area for further research and could be a valuable predictor of serviceuse and a worthwhile factor to explore related to the concept of equity of access.

\section{Conclusion}

As more people survive initial trauma and reintegrate into the community, significant implications arise to address long-term service need, access, use, planning and factors associated with these and with an optimal quality of life. At the same time an aging population and fiscal constraints for health services require an openness to explore the benefits of varied models, including $\mathrm{CM}$ approaches that address individual characteristics, such as those examined in this study. The formative role of the case manager in assessing service needs and in enabling access to services through education and identification of risk factors, combined with the plurality of services available to persons following TBI, underscores the need to further understand the relationship between CM and TBI. The Life Care Planner has the unique ability to devise plans and recommend supports using a combined case management model and health service use model, with consideration of science-based predictors. This Canadian research study elucidates the multi-factorial features in predicting service use, and the potential use of scientific models by professionals in the health service roles of CM and LCP. 


\section{Acknowledgments}

The authors are grateful for the support of the Ontario Brain Injury Association for making the data available and for the help of Mr. John Dumas for his assistance in analysis and interpretation. Drs. Kathy Boschen and Dorcas Beaton are thanked for their input into the analyses, interpretation, and reviews of earlier drafts of this work.

\section{References}

Abreu, B. C., Seale, G., Scheibel, R. S., Huddleston, M. S., Zhang, L., \& Ottenbacher, K. J. (2001). Levels of self-awareness after acute brain injury: How patients' and rehabilitation specialists' perceptions compare. Archives of Physical Medicine and Rehabilitation, $82,49-56$.

Aday, L. A., \& Andersen, R. (1974). A framework for the study of access to medical care. Health Services Research, 9, 208-220.

Andersen, R. M. (1995). Revisiting the behavioral model and access to medical care: Does it matter? Journal of Health and Social Behavior, 36, 1-10.

Andersen, R. M., \& Davidson, P. L. (2001) Improving access to care in America: Individual and contextual indicators. In R. M. Andersen, T. H. Rice, G. F. Kominski (Eds). Changing the U.S. healthcare system: Key issues in health services policy and management, (pp. 3-30). San Francisco: Jossey-Bass.

Andersen, R. M., Davidson, P. L., \& Ganz, P. A. (1994). Symbiotic relationships of quality of life, health services research and other health research. Quality of Life Research, 3, 365-371.

Applebaum, R., \& White, M. (2000). Key issues in case management around the globe. San Francisco: American Society on Aging.

Bland, D. C., Zampieri, C., \& Damiano, D. L. (2011). Effectiveness of physical therapy for improving gait and balance in individuals with traumatic brain injury: A systematic review. Brain Injury, 25(7-8), 664-679.

Chen, A. Y., Zagorski, B., Parsons, D., Vander Laan, R., Chan, V., \& Colantonio, A. (2012). Factors associated with discharge destination from acute care after acquired brain injury in Ontario, Canada. BMC Neurology, 12-16.

Cochrane, J., Goering, P., Durbin, J., Butterill, D., Dumas, J., \& Wasylenki, D. (2000) Tertiary mental health services: II. Subpopulations and best practices for service delivery. Canadian Journal of Psychiatry, 45, 186-190.

Cohen, M. M., Noralou, P. R., DeCoster, C., Black, C., \& Decker, K. M. (1995) Manitoba's population-based databases and long-term planning: Beyond the hospital databases. Health Care Management Forum, 8, 5-13.

Deutsch, P., \& Sawyer, H. (1985-2007). A guide to rehabilitation. White Plains: Ahab Press.

Drag, L., Renninger, C., King, R., \& Hoblyn, J. (2013). Predictors of inpatient and outpatient healthcare utilization in veterans with traumatic brain injury. Journal of Head Trauma Rehabilitation, 28(1), 39-47.

Fitzsimmons, R. D. (2003) Brain injury case management: The potential and limitations of late-stage intervention-a pilot study. Brain Inj, 11, 947-971.
Forbes, D. A., \& Janzen, B. L. (2004) Comparison of rural and urban users and non-users of homecare in Canada. Canadian Journal of Rural Medicine, 9, 227-235.

Goldsmith, L. (2002). A critical history of Andersen's Behavioral Model of Health Services Use: A reflection of how we study access to health care. Abstr Acad Health Serv Res Health Policy Meet, 9,6 .

Heinemann, A. W., Corrigan, J. D., \& Moore, D. (2004). Case management for traumatic brain injury survivors with alcohol problems. Rehabilitation Psychology, 49, 156-166.

Heinemann, A. W., Sokol, K., Garvin, L., \& Bode, R. K. (2002) Measuring unmet needs and services among persons with traumatic brain injury. Archives of Physical Medicine and Rehabilitation, 83, 1052-1059.

Hodgkinson, A., Veerabansa, A., Drane, D., \& McCluskey, A. (2000). Service utilization following traumatic brain injury. Journal of Head Trauma Rehabilitation, 15, 1208-1226.

Hosmer, D. W., \& Lemeshow, S. (2000). Applied logistic regression, New York: John Wiley \& Sons Inc.

Klinger, L., Baptiste, B., \& Adams, J. R. (2004). Life care plans: An emerging area for occupational therapists. Can J Occup Ther, 71(2) 88-99.

Kraus, J. F., \& Chu, L. D. (2005). Epidemiology. In J. M. Silver, T. W. McAllister, \& S. C. Yudofsky (Eds.), Textbook of traumatic brain injury, (pp. 3-26). Washington D.C.: American Psychiatric Publishing, Inc.

Laursen, B., \& Helweg-Larsen, K. (2012). Health service use in adults $20-64$ years with traumatic brain injury, spinal cord injury or pelvic fracture. A cohort study with 9-year follow-up. BMJ Open, 2. doi: 10.1136/bmjopen-2012-001521

Masel, B. E., \& DeWitt, D. S. (2010). Traumatic brain injury: A disease process, not an event. Journal of Neurotrauma, 27, 1529-1540.

McColl, M. A. H., Shortt, S., Hunter, D., Dorland, J., Godwin, M., Rosser, W., \& Shaw, R. (2010). Access and quality of primary care for people with disabilities: A comparison of practice factors. Journal of Disability Policy Studies, 21(3), 131-140.

National Case Management Network (2009). Canadian Standards of Practice for Case Management. Toronto, ON: National Case Management Network. Retrieved from http://www.ncmn.ca/resources/documents/english\%20standards $\% 20$ for\%20web.pdf

Ontario Brain Injury Association (2013). Annual report. St. Catharines, ON. Retrieved from http://obia.ca/wp-content/ uploads/2013/01/2013-Annual-Report_final.pdf

Ontario Brain Injury Association (2012). The OBIA Impact Report 2012: A statistical snapshot of acquired brain injury and its effects on survivors and caregivers. St. Catharines, ON: Ontario Brain Injury Association.

Ouellet, M. C., Sirois, M. J., \& Lavoie, A. (2009). Perceived mental health and needs for mental health services following trauma with and without brain injury. Journal of Rehabilitation Medicine, 41(3), 179-186.

Phillips, V. L., Greenspan, A. I., Stringer, A. Y., Stoble, A. K., \& Lehtonen, S. (2004). Severity of injury and service utilization following traumatic brain injury. Journal of Head Trauma Rehabilitation, 19, 217-225.

Public Health Agency of Canada and National Health Charities of Canada (2014). Mapping Connections: An understanding of neu- 
rological conditions in Canada. National Population Health Study of Neurological Conditions. Ottawa, ON: Public Health Agency of Canada.

Quinn, J., Pannone, P., Gruman, C., \& Roja, M. (2004). Long-term care insurance: Care management practice issues. Care Manag $J$, $5(1), 25-33$.

Schootman, M., \& Fuortes, L. (1999). Functional status following traumatic brain injuries: Population-based rural-urban differences. Brain Injury, 13, 995-1004.

Svenson, J. E., \& Spurlock, C. W. (2001). Insurance status and admission to hospital for head injuries: Are we part of a two-tiered medical system? Am J Emerg Med, 19(1), 19-24.

Tabachnick, B. G., \& Fidell, L.S. (2001). Using multivariate analysis. Boston: Allyn and Bacon.

Tennant, A., MacDermott, N., \& Neary, D. (1995). The long-term outcome of head injury: Implications for service planning. Brain Injury, 9, 595-605.
Tomiak, M., Berthelot, J. M., Guimond, E., \& Mustard, C. A. (2000). Factors associated with nursing-home entry for elders in Manitoba, Canada. Journal of Gerontology, 55A, M279-M287.

Van Holst, H., \& Cassidy, J. D. (2004). Mandate of the WHO collaborating centre task force on mild traumatic brain injury. Journal of Rehabilitation Medicine, 43, 8-10.

Weed, R. O. \& Berens, D. E. (2010). Life care planning: Past, present, and future. In R. O. Weed, \& D. E. Berens (Eds.), Life care planning and case management handbook, (3rd Ed., pp. 1-13), Boca Raton, FL: CRC Press.

Willemse-van Son, A. H., Ribbers, G. M., Stam, H. J., \& Van den Bos, G. A. (2009). Is there equity in long-term healthcare utilization after traumatic brain injury? Journal of Rehabilitation Medicine, 41(1), 59-65.

World Health Organization (2014). International classification of functioning, disability and health. Geneva, SW. Retrieved from http://www.who.int/classifications/icf/en/ 